\title{
SEEING SMELLS: DEVELOPMENT OF AN OPTOELECTRONIC NOSE $\#$
}

Kenneth S. Suslick*, Daniel P. Bailey, Crystal K. Ingison, Michael Janzen, Margaret E. Kosal, William B. McNamara III, Neal A. Rakow, Avijit Sen, Jeremy J. Weaver, Jennifer B. Wilson, Chen Zhang, and Shirley Nakagaki Department of Chemistry, University of Illinois at Urbana-Champaign, 600 S. Mathews Av., Urbana, IL 61801, USA

Recebido em 7/6/06; publicado na web em 26/3/07

\begin{abstract}
The development of an array of chemically-responsive dyes on a porous membrane and in its use as a general sensor for odors and volatile organic compounds (VOCs) is reviewed. These colorimetric sensor arrays (CSA) act as an "optoelectronic nose" by using an array of multiple dyes whose color changes are based on the full range of intermolecular interactions. The CSA is digitally imaged before and after exposure and the resulting difference map provides a digital fingerprint for any VOC or mixture of odorants. The result is an enormous increase in discriminatory power among odorants compared to prior electronic nose technologies. For the detection of biologically important analytes, including amines, carboxylic acids, and thiols, high sensitivities (ppbv) have been demonstrated. The array is essentially non-responsive to changes in humidity due to the hydrophobicity of the dyes and membrane.
\end{abstract}

Keywords: electronic nose; metalloporphyrins; sensor technology.

\section{INTRODUCTION}

The detection of volatile organic compounds (VOCs) is generally done either by a separation and component identification (e.g., GC, GC-MS) or by a holistic response of a general sensor array (i.e., "electronic nose"). While gas chromatography is fully mature, electronic nose technology remains at a relatively primitive level. Such devices have generally used multiple, cross-reactive sensors based primarily on changes in properties of some set of polymers (e.g., mass, volume, conductivity) or on electrochemical oxidations at a set of heated metal oxides ${ }^{1,2}$ Most prior electronic nose technology suffers from some severe limitations, however: the detection of compounds at low concentrations relative to their vapor pressures is difficult; the discrimination between compounds within a similar chemical class has been limited; and perhaps most importantly, interference from the large environmental changes in humidity remains problematic.

In recent years, we have developed a rather different, but quite simple, approach to an "optoelectronic nose" based on the colorimetric array detection of a wide range of odorants initially using metalloporphyrins ${ }^{3-5}$ and more generally chemically-responsive dyes $^{6-12}$. The importance of including metal ion containing sensors in such an array is confirmed by the recent indications that the mammalian olfactory receptors are in fact metalloproteins ${ }^{13}$. Here we review the development of our colorimetric sensor array and demonstrate its success in addressing the current problems with electronic nose technology.

\section{THE COLORIMETRIC SENSOR ARRAY}

The detection and identification of chemicals is a fundamental aspect of the area of supramolecular chemistry known as "molecular recognition". Intrinsically, such processes involve the interactions between molecules. The classification of inter-molecular interactions is well established (Figure 1) and involves bond

\footnotetext{
* e-mail: ksuslick@uiuc.edu

\# presented at the $29^{\text {th }}$ Reunião Anual da Sociedade Brasileira de Química as invited conference

•Depto. de Química - Universidade Federal do Paraná
}

formation and coordination, acid-base interactions, hydrogenbonding, charge-transfer and $\pi-\pi$ molecular complexation, dipolar and multipolar interactions, and last and least, van der Waals interaction and physical adsorption.

Remarkably, most prior electronic nose technology and nearly all chromatographic separation techniques rely essentially exclusively on van der Waals and physical adsorption, the weakest and least selective of forces between molecules. We believe this to be a fundamental flaw in the development of sensors with both high sensitivity and high selectivity. In many ways, our colorimetric sensor array revisits the earlier, pre-electronic era of analytical chemistry ${ }^{14}$, updated by the addition of modern digital imaging and pattern recognition techniques.

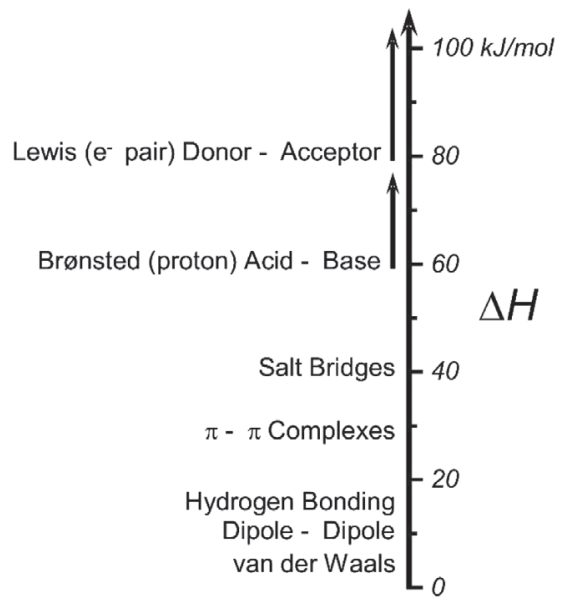

Figure 1. Classes of intermolecular interactions and their approximate heats of enthalpy

In addition, the development of new sensor technology faces the dilemma of trying to create sensors that are both increasingly sensitive and increasingly robust. One may argue that beyond a certain point, the more sensitive a sensor becomes, inherently the less robust it can be. The path around this dilemma is the development of disposable sensors, thus unlinking the opposing 
demands. Colorimetric sensor arrays provide one successful method of doing so.

There are two fundamental design requirements for a colorimetric sensor array: (1) the chemo-responsive dye must contain a center to interact strongly with analytes, and (2) this interaction center must be strongly coupled to an intense chromophore. The first requirement implies that the interaction must not be simple physical adsorption, but rather must involve other, stronger chemical interactions. Chemoresponsive dyes are those dyes that change color, in either reflected or absorbed light, upon changes in their chemical environment. The consequent dye classes from these requirements are (1) Lewis acid/base dyes (i.e., metal ion containing dyes), (2) Brønsted acidic or basic dyes (i.e., $\mathrm{pH}$ indicators), and (3) dyes with large permanent dipoles (i.e., zwitterionic solvatochromic dyes) (Figures 2 and 3).

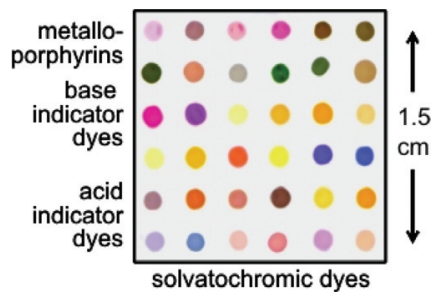

Figure 2. A colorimetric sensor array containing 36 chemically responsive dyes. Arrays can be uniformly prepared by non-contact printing on a variety of inert, hydrophobic substrates

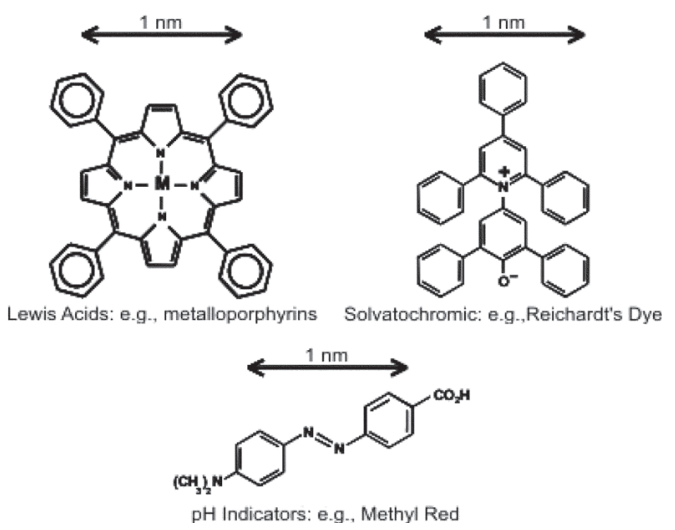

Figure 3. The components of colorimetric sensor arrays are inherently nanoscale. The 36 dyes were selected empirically based on the quality of their color response to a representative selection of different analytes

The dyes that are used in the CSA are all commercially available $^{15}$ (as are the arrays from ChemSensing, Inc., Champaign, IL USA; www.chemsensing.com) The use of porphyrins and their metal complexes are a natural choice for recognition of analytes with Lewis acid/base capabilities. Metalloporphyrins are nearly ideal for the detection of metal-ligating vapors because of their open coordination sites for axial ligation, their large spectral shifts upon ligand binding, and their intense coloration. A series of metalated tetraarylporphyrins was used to provide differentiation based on metal-selective coordination. Common $\mathrm{pH}$ indicator dyes change color in response to changes in the proton (Brønsted) acidity or basicity of their environment. Solvatochromic dyes change color in response to changes in the general polarity of their environment, primarily through strong dipole-dipole interactions ${ }^{16}$. To some extent, all dyes inherently are solvatochromic, although some are much more responsive than others; among the most responsive are Reichardt's Dye and Nile Red.

\section{DISCRIMINATION OF VOCS}

In some ways, the colorimetric sensor array is simply a digitally imaged two-dimensional extension of litmus paper. For any volatile analyte, odorant, or complex mixture of odorants, a difference map is easily generated by digital subtraction, pixel by pixel, of the image of the array before and after exposure: red value after exposure minus red value before, green minus green, blue minus blue. Averaging of the centers of the spots ( 200 pixels) avoids artifacts from nonuniformity of the dye spots, especially at their edges. Although the resulting data is inherently digital (simply a vector of $3 \mathrm{~N}$ dimensions where $\mathrm{N}=$ total number of spots), the difference map is conveniently visualized as the same array of dyes whose color values are the absolute values of the differences (as shown in Figure 4).
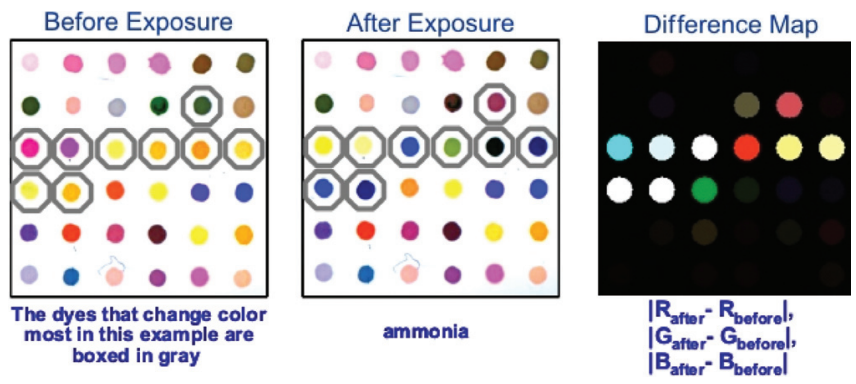

Figure 4. The difference map of a colorimetric array can be generated for any odorant by digital subtraction, pixel by pixel, of the image of the array before and after exposure. In the difference maps, the center 300 pixels of each spot are averaged to avoid edge artifacts

Sensor array responses were determined for a series of 100 different VOCs representing the common organic functionalities ${ }^{10}$ : amines, arenes, alcohols, aldehydes, carboxylic acids, esters, halocarbons, ketones, phosphines, sulfides, and thiols. These patterns are shown in Figure 5 for a 36 dye array. Excellent discrimination among these analytes is observed even without any statistical analysis.

Each analyte response is actually represented digitally as the change in the red, green, and blue values of each of the 36 dyes, i.e., a 108 dimensional vector. A principal component analysis ${ }^{17}$ (PCA) of the full set of the VOC patterns reveals a surprisingly high degree of dispersion among the independent dimensions created by linear combinations of the RGB responses of the 36 dyes used in these arrays. The PCA shows that $95 \%$ of the discriminatory range requires 22 dimensions. This extremely high dispersion reflects the very wide range of chemical-properties space being probed by our choice of chemoresponsive dyes. In comparison, most prior electronic nose technology is dominated by only a two or three independent dimensions (one of which, hydrophobicity, often accounts for $90 \%$ of the discrimination $)^{1,2}$; this is the inherent result of relying on van der Waals and other weak interactions for molecular recognition.

With such a high dimensionality, the usual "score plots" of two or three dimensions that are commonly generated from PCA are of very limited value for analysis of CSA data. Instead, we must use a technique that utilizes the full dimensionality of the data. Hierarchical Cluster Analysis (HCA) does this by systematically examining the distance between the vectors that represent each odorant in this 108dimensional RGB color space. HCA makes use of only the digital data representing the observed difference maps ${ }^{17}$ - no chemical information is included. A dendrogram is generated that shows the clustering of the data, much like an ancestral tree. The resulting dendrogram for the responses to saturated analyte vapors is shown in Figure 6. Remarkably, the clusters formed are in keeping with the 


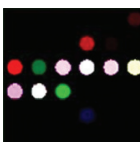

decylamine

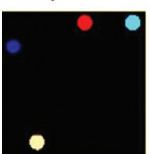

hexanethiol

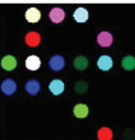

$\mathrm{PhMe}_{2}$ phospine

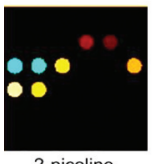

2-picoline

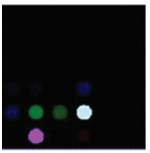

pentanol

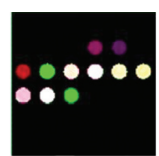

sec-Bu $\mathrm{Bumine}_{2}$

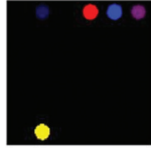

benzylthiol

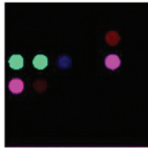

$\mathrm{Bu}_{3}$ phosphine

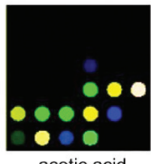

eetic acid

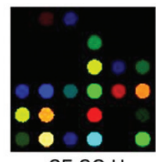

$\mathrm{CF}_{3} \mathrm{CO}_{2} \mathrm{H}$

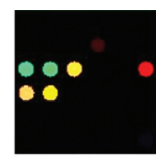

aniline

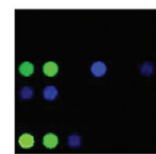

ethanol
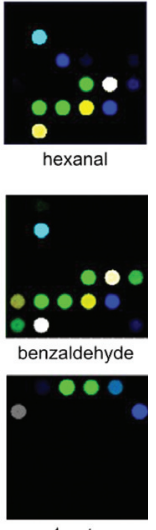

1-octene
Figure 5. Colorimetric array responses for some common volatile organic compounds at full vapor pressure at 300K. Every VOC has a different pattern, while at the same time the patterns reflect familial resemblances (e.g., amines are similar to one another, acids are similar, aldehydes are similar, etc.)

qualitative structural and electronic properties of the VOCs. The familial similarities among compounds of the same functionality are exceptional: amines, alcohols, aldehydes, esters, etc. are all easily distinguished from each other. This also means that once a library is established, an unknown odorant can be classified by comparison and one can then determine what this new odorant is "like".

The discriminatory power of the CSA is enormous and even very subtle differences between analytes are easily detected. With a 36dye array, each of the 108 dimensions (i.e., 36 RGB's) can take on one of 256 possible values (for inexpensive 8 bit scanners or digital cameras). The theoretical limit of discrimination, then, would be the number of possible patterns, i.e., $(256)^{108}$. Realistically, however, the RGB vector components do not range over the full 256 possible values; we do observe R, G, and B values vary over a range of 40 . To discriminate patterns, let us assume a change of at least 4 is needed in the R, G, or B value (we can actually easily discriminate with changes of 2). From the PCA, not all of the 108 dimensions are equally important. In fact, roughly $95 \%$ of all information is contained in $\sim 22$ specific dimensions (i.e., linear combinations of the 108 different R, G, and B values). This implies a 'practical' limit of discrimination that is still immensely large: $(40 / 4)^{22}=10^{22}$ distinct patterns should be recognizable in a 36-dye colorimetric sensor array.

\section{INTERFERENCE FROM HUMIDITY}

Physisorption of molecules on surfaces is dominated by the relative hydrophobicity of the adsorbate and adsorbent. It should be no surprise, therefore, that a serious weakness in current electronic nose technology is sensitivity to changes in humidity. Water vapor ranges in the environment from $<2000$ to $>20,000$ ppmv; if one is interested in concentrations of VOCs that are tens of ppbv, or even few ppmv, even a very low level of interference from water is simply unacceptable.

For our colorimetric sensor arrays, both the dyes and the membrane on which they are printed have been selected to be highly hydrophobic. In fact, they can even be used by direct immersion in water to identify and quantify low concentrations of dissolved organic compounds ${ }^{12}$. As a consequence, these arrays are impervious to changes in relative humidity. As shown in Figure 7, the dyes are essentially unresponsive to water vapor. The water-vapor insensitivity of our technology provides a substantial advantage in the analysis of real-world samples.

\section{SENSITIVITY}

Our colorimetric array sensor technology is based on strong and relatively specific interactions between the analytes and a chemically responsive dye library. In prior electronic nose technology, the use of

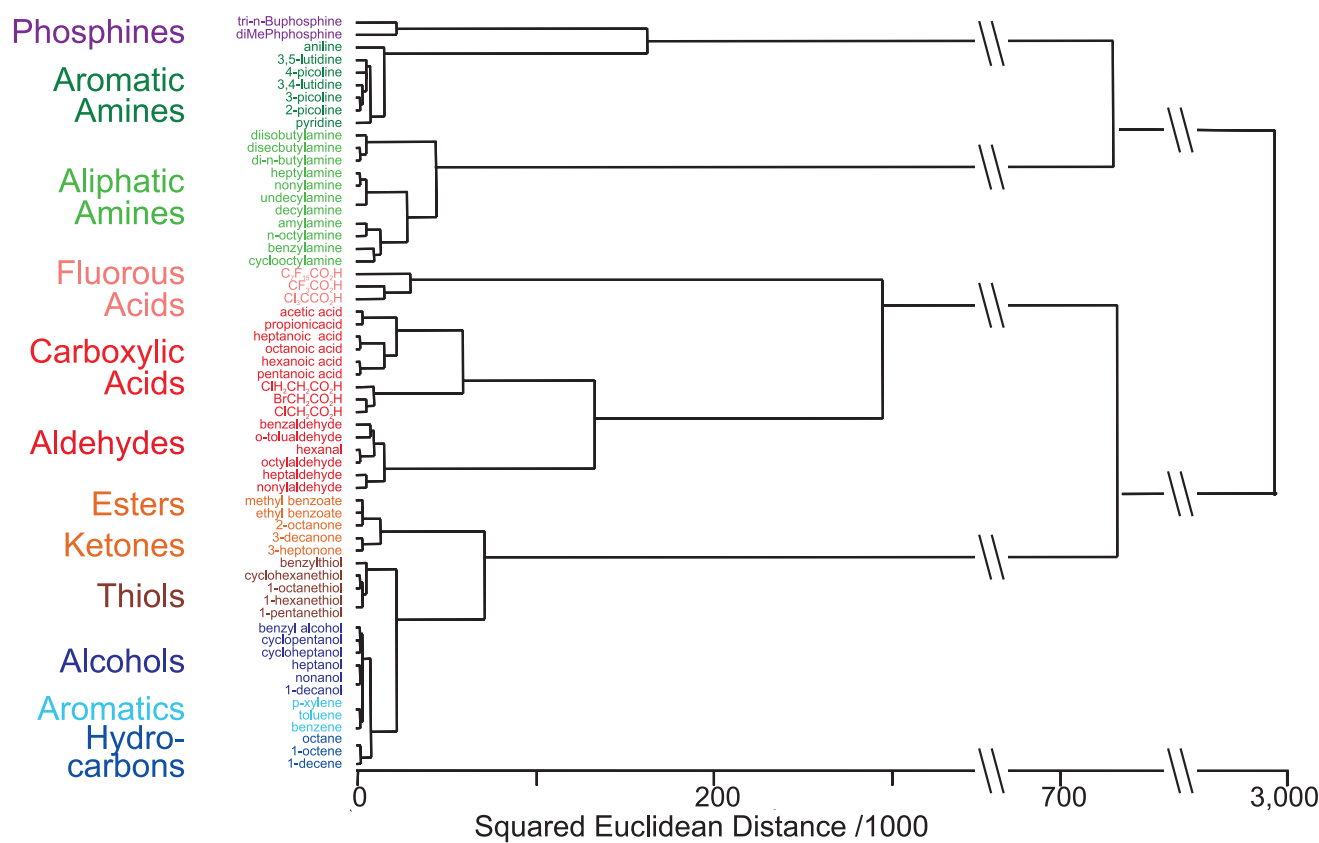

Figure 6. Hierarchical Cluster Analysis Dendrogram of the colorimetric array responses to 32 common organic compounds at full vapor pressure at $300 \mathrm{~K}$ 


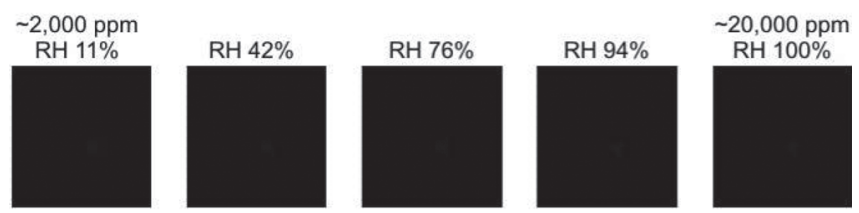

Figure 7. Difference maps upon exposure to varying relative humidity $(R H)$. The colorimetric array is insensitive to changes in $R H$. Similarly, changes in humidity do not significantly affect the color fingerprints of other analytes

adsorption into polymer arrays (e.g., conductive polymer arrays, quartz microbalance or surface acoustic wave detectors coated with a variety of polymers, or polymers doped with single indicating fluorophore) depends upon weak matrix-analyte interactions to provide limited selectivity and relatively poor sensitivity. A major advantage of our sensor arrays is that they are able to provide unique patterns for the identification of odors even at extremely low vapor concentrations of many analytes because we rely on strong interactions between analyte and sensor dyes for molecular recognition.

Metal-ligand (i.e., metal-analyte) bonds range in their bond enthalpies from $\sim 40$ to $\sim 200 \mathrm{~kJ} / \mathrm{mol}$. In non-coordinating solvents (e.g., alkanes), equilibrium binding constants are often $>10^{6} \mathrm{M}^{-1}$. For pyridine, the vapor pressure is $0.02 \mathrm{~atm}$ at room temperature, so we have a Raoult's constant of $\sim 2 \times 10^{-3} \mathrm{~atm} \mathrm{M}^{-1}$. For a binding constant of $\sim 10^{6} \mathrm{M}^{-1}$, this is equivalent to $\sim 2 \mathrm{ppbv}$ vapor. In contrast, the enthalpy of physical adsorption (e.g., into polymers) is only $\sim 5$ to $20 \mathrm{~kJ} / \mathrm{mol}$ (i.e., roughly a tenth of a metal bond). Therefore, the equilibrium constant for adsorption will typically be only about 5 x $10^{-5}$ as large as that for ligation to metal ions. Therefore, ligation is intrinsically $\sim 20,000$-fold more sensitive than adsorption into polymers. Differences in the sensitivity of detection techniques, of course, can either enhance or diminish this intrinsic advantage of ligation and other strong interactions over physical adsorption and other weak interactions (e.g., van der Waals).

As shown in Figure 8, this expectation of improved sensitivities is realized experimentally. Even GC-MS achieves typically only ppmv to 100 ppbv sensitivities for VOCs in the absence of pre-concentration. Sub-ppmv discrimination is not a problem with our colorimetric array detection for functionalized analytes such as amines, carboxylic acids, phosphines and thiols. In fact, we can extend our sensitivities down to the low ppbv regime for many such analytes. Extension of sensitivity by improved imaging technology (e.g., true 16-bit or 24bit color resolution in place of our currently used 8-bit scanners) may improve our sensitivity by a thousand-fold.

For quantitative analysis of analyte concentrations with colorimetric arrays, every analyte at a different concentration is best thought of as a different analyte. For each analyte, a specific dye will begin to change color at some concentration and as the analyte concentration increases, the color change will asymptotically saturate; some dyes change at low concentrations of any given analyte, some only at very high concentrations. Quantitative analysis of single analytes (or of a single analyte changing in a constant background) can be easily done by comparison to a pattern library of analyte concentration.

While our colorimetric sensor arrays are disposable, this does not mean that they are not reusable. The CSA is best through of as a "chemical fuse": just as with an electric fuse, as long as the concentration of the odorant (i.e., the current) fluctuates within some range, the CSA (fuse) is unaffected. But, if the concentration increases to too high a value, the CSA will take too long to recover: i.e., the fuse is blown and the CSA should be replaced. As shown by one example in Figure 9, the CSA will reproducibly cycle between the PEL and IDLH concentrations of many toxic industrial chemicals.

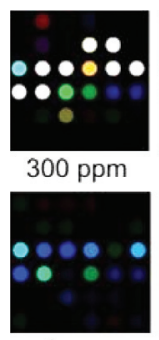

$1 \mathrm{ppm}$

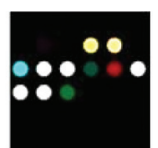

$50 \mathrm{ppm}$

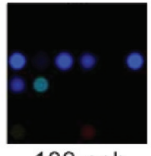

$100 \mathrm{ppb}$
$10 \mathrm{ppb}$

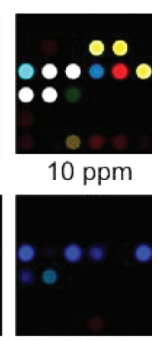

Figure 8. Colorimetric array sensitivity has limits of recognition are well below 1 ppmv for most TICs. Array difference maps are shown for ammonia as a function of concentration. The limit of detection is $<10 \mathrm{ppbv}$; for comparison, the immediately dangerous to life or health level (IDLH) and the permissible exposure level (PEL) levels for ammonia are 300 and 50 ppmv, respectively

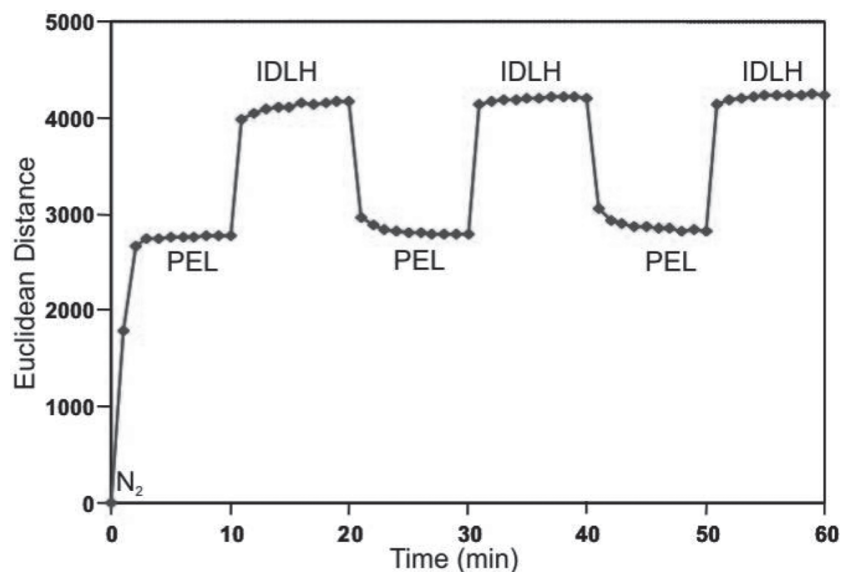

Figure 9. Exposure of a fresh array under $\mathrm{N}_{2}$ to repeated cycling of $\mathrm{SO}_{2}$ at its PEL (5 ppmv) to $\mathrm{SO}_{2}$ at IDLH (100 ppmv). Data acquired every min.; equilibrated response time is $2 \mathrm{~min}$

\section{ANALYSIS OF COMPLEX MIXTURES: BEER}

Chemists have a natural tendency to assume that for any complex mixture, the single analytical goal is a complete component-bycomponent analysis. But in fact, multiple analytic goals for complex mixtures are conceivable: comparison to a standard (e.g., quality control, counterfeit detection), identification of chemical class or family of an unknown VOC, correlation of odors to properties (e.g., "good smelling" as determined by a human sensory evaluation panel), changes in concentrations of a few components against a constant background, and of course, a complete component by component (CBC) analysis. Separation techniques (e.g., GC, HPLC, CE, etc.) excel at CBC analyses. For many other applications, however, a composite response may be not only sufficient, but actually preferred. For this reason, the mammalian olfactory system (and likewise, electronic nose techniques, in general), give a composite response to complex mixtures.

As a closing example of the application of our CSA, we have soberly examined eighteen commercial beers in both liquid and gas phases using colorimetric sensor arrays with hydrophobic dyes printed on a hydrophobic membrane ${ }^{11}$. Digital imaging of the dye array before and after exposure to the complex analytes in either the liquid-phase or the head-gas provides a color change profile as a unique fingerprint for the specific analyte. In both liquid and gas phase experiments, facile identification for each of the analytes was achieved by eye or more quantitatively, by statistical analyses. The HCA dendrogram of these beer trials are shown in Figure 10. Substantial improvement in analyte discrimination was obtained via the combination of the 


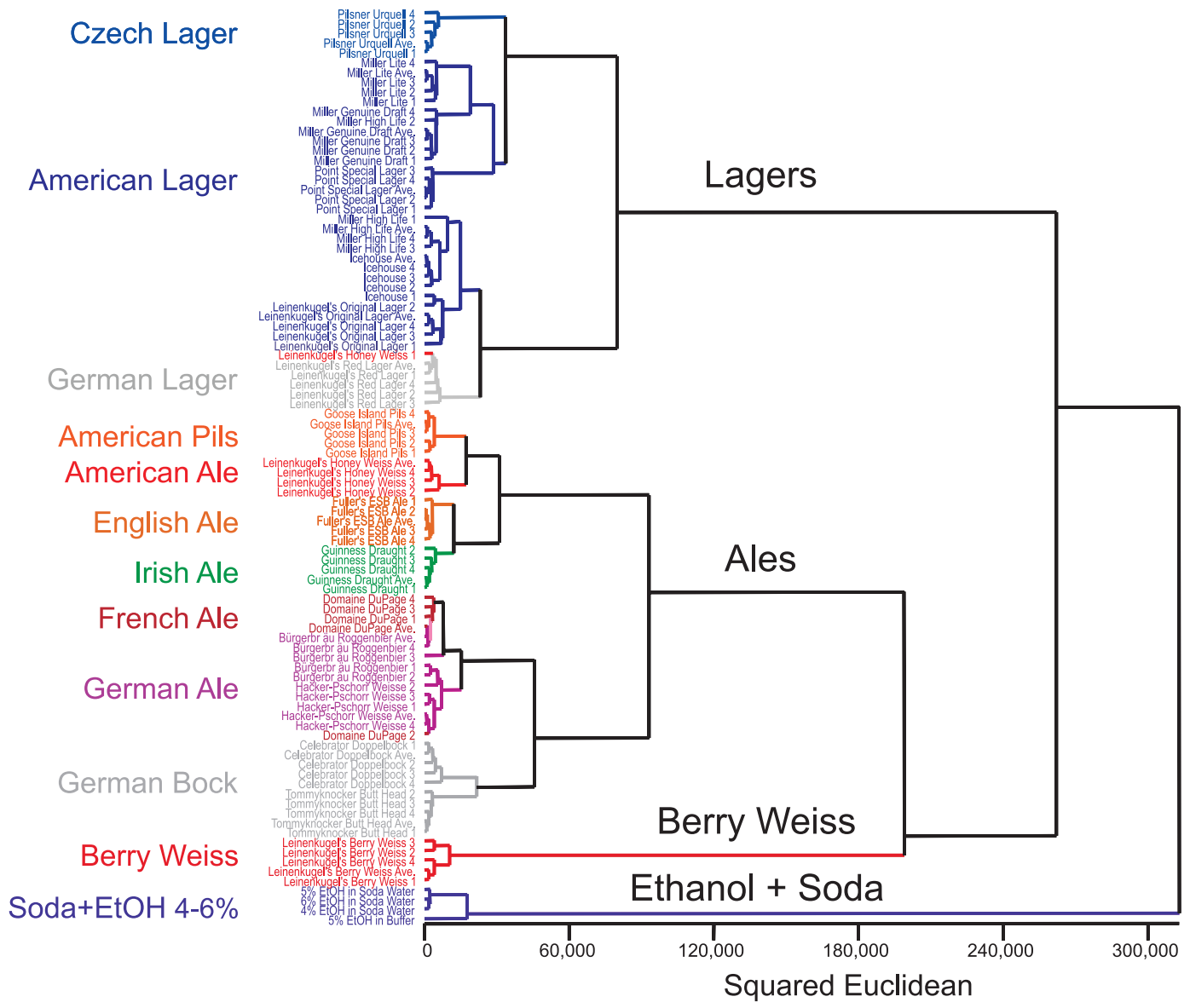

Figure 10. A dendrogram of 94 tests of 18 beers. Clear separation of beers by class (i.e., lager vs. ale) is observed and even very similar beers are distinguishable

digital databases for each of the two phases, with classification errors about 3\% (3 misidentifications out of 94 cases).

The high level of discrimination that we observe in the analysis of different beers suggests that the colorimetric sensor array will also be able to distinguish readily between a beer in its pristine state and one that has undergone spoilage, has been foxed by overheating or photo-oxidation, has been watered or contaminated, or has loss carbonation. In addition, the extremely high dimensionality of the data from our colorimetric arrays allows statistical correlations with the evaluations by organoleptic panels (i.e., drinkers) that may prove to have quantitative predictive value.

\section{ACKNOWLEDGMENTS}

This work was supported by the U.S. NSF and HSARPA and in part by the DOE Seitz Materials Research Laboratory under Grant DEFG02-91-ER45439. K.S.S. and S.N. are particularly grateful to the Sociedade Brasileira de Quimica for providing the opportunity to prepare this paper in the most charming of environments. Finally, we would like to acknowledge the efforts of staff, past and present of ChemSensing, Inc., who have contributed in many ways to the development of this technology; for purposes of full disclosure, K. S. Suslick acknowledges that he is a Director of and has a significant financial interest in ChemSensing, Inc.

\section{REFERENCES}

1. Albert, K. J.; Lewis, N. S.; Schauer, C. L.; Sotzing, G. A.; Stitzel, S. E.; Vaid, T. P.; Walt, D. R. Chem. Rev. 2000, 100, 2595; Deisingh, A. K.; Stone, D. C.; Thompson, M. Int. J. Food Sci. Technol. 2004, 39, 587.

2. Gardner, J. W.; Bartlett, P. N.; Electronic Noses: Principles and Applications, Oxford University Press: New York, 1999; Toko, K.; Biomimetic Sensor Technology, Cambridge University Press: Cambridge, UK, 2000.

3. Rakow, N. A.; Suslick, K. S.; Nature 2000, 406, 710.

4. Rakow, N. A.; Suslick, K. S. In Artificial Chemical Sensing: Olfaction and the Electronic Nose; Stetter, J. R.; Pensrose, W. R., eds.; Electrochem. Soc.: Pennington, 2001, p. 8-14.

5. Suslick, K.S.; Rakow, N. A.; U.S. Patent 6,368,558 2002

6. Suslick, K. S.; Rakow, N. A.; Sen, A. U.S. Patent 6,495,102 B1 2002

7. Rakow, N. A.; Sen, A.; Janzen, M. C.; Ponder, J. B.; Suslick, K. S.; Angew. Chem. Int. Ed. 2005, 44, 4528 .

8. Suslick, K. S.; Rakow, N. A.; Sen, A.; Tetrahedron 2004, 60, 11133.

9. Suslick, K. S.; MRS Bull. 2004, 29, 720.

10. Janzen, M. C.; Ponder, J. B.; Bailey, D. P.; Ingison, C. K.; Suslick, K. S.; Anal. Chem. 2006, 78, 3591.

11. Zhang, C.; Bailey, D. P.; Suslick, K. S.; J. Agric. Food Sci. 2006, 54, 4925

12. Zhang, C.; Suslick, K. S; J. Am. Chem. Soc 2005, 127, 11548.

13. Wang, J.; Luthey-Schulten, Z.; Suslick, K. S.; Proc. Natl. Acad. Sci. U.S.A. 2003, 100, 3035

14. Kolthoff, I. M.; Acid Base Indicators, Rosenblum, C., trans.; Macmillan: New York, 1937.

15. Green, F. J.; The Sigma-Aldrich Handbook of Stains, Dyes and Indicators, Aldrich Chemical Company, Inc, 1990, p. 776.

16. Reichardt, C.; Chem. Rev. 1994, 94, 2319.

17. Haswell, S. J.; Practical Guide To Chemometrics, Dekker: New York, 1992, p. 324. 